\title{
Long-Term Follow-Up of Microsurgical Reconstruction for Pelvic Tumor Focusing on Spinal Deformity and Quality of Life
}

\author{
Soichi Ejiri*, Shin-ichi Kikuchi, Takahiro Tajino, Ryoichi Kawakami, Michiyuki Hakozaki, \\ Shin-ichi Konno \\ Department of Orthopaedic Surgery, Fukushima Medical University School of Medicine, Fukushima, Japan \\ Email: sejiri@fmu.ac.jp
}

Received 20 February 2014; revised 16 March 2014; accepted 23 March 2014

Copyright () 2014 by authors and Scientific Research Publishing Inc.

This work is licensed under the Creative Commons Attribution International License (CC BY).

http://creativecommons.org/licenses/by/4.0/

(c) () Open Access

\begin{abstract}
Background: To provide patients with a superior quality of life (QOL) after their pelvic tumor resection, ensuring the stability of the trunk as well as satisfactory lower extremity function is important. Although microsurgical reconstructions for the pelvic ring and hip are used clinically, the details of postoperative long-term spinal deformity and QOL remain unclear. Methods: The patients were 66- and 43-year-old men and a 43-year-old woman. The mean postoperative follow-up period was 134 months. The surgical procedures performed on these patients were: pelvic ring reconstruction using a double-barreled free vascularized fibular graft (FVFG) in Patient 1; simultaneous pelvic ring reconstruction using FVFG and hip arthrodesis in Patient 2; and latissimus dorsi free flap without pelvic ring reconstruction in Patient 3 . As indicators of spinal deformity, we measured the Cobb angle, thoracic kyphosis angle, lumbar lordosis angle, pelvic angle on the sagittal plane, and sagittal plane balance using whole spine radiography. To assess the patients' QOL, we examined the International Society of Limb Salvage (ISOLS) score, the Roland-Morris Disability Questionnaire (RDQ), activities of daily living (ADL) satisfaction using a visual analogue scale (VAS), and the SF-36. Results: Spinal alignment in the frontal plane worsened in order from Patient 1 to 3. Spinal alignment and pelvic tilt in the sagittal plane were at appropriate levels in Patients 1 and 2 . The trunk tilt in the sagittal plane was at an appropriate level only in Patient 1 . In the QOL assessment, the function of the affected limb worsened in order from Patient 1 to 3 . RDQ scores were lower than the national norm in Patients 2 and 3. With respect to ADL satisfaction and the SF-36, Patient 3 had a markedly low VAS, physical functioning, role-physical, and role-emotional scores. Conclusion: In the long-term clinical course after pelvic tumor resection, in order to obtain satisfactory spinal alignment and QOL, pelvic ring reconstruction and hip arthrodesis are important.
\end{abstract}

\footnotetext{
${ }^{*}$ Corresponding author.
}

How to cite this paper: Ejiri, S., et al. (2014) Long-Term Follow-Up of Microsurgical Reconstruction for Pelvic Tumor Focusing on Spinal Deformity and Quality of Life. Journal of Cancer Therapy, 5, 354-362. 


\section{Keywords}

\section{Pelvic Tumor, Pelvic Ring Reconstruction, Quality of Life, Spinal Deformity, Vascularized Fibular Graft}

\section{Introduction}

After an individual undergoes a pelvic tumor resection, it is important that he or she achieves adequate trunk support as well as satisfactory lower extremity function in order to gain an adequate quality of life (QOL) [1]. Pelvic ring reconstruction and hip arthrodesis using a microsurgical technique are reported to have superior treatment outcomes [1]. However, no detailed reports have investigated the differences in long-term ( $\geq$ postoperative 10 years) spinal deformity and QOL caused by different extents of bone resection or reconstructive procedures. In the present study therefore, we investigated the cases of three patients who underwent different reconstructive surgeries that used microsurgery after pelvic tumor resection $\geq 10$ years ago, and we evaluated the effects of reconstructed pelvic ring and hip function on spinal deformity and QOL.

\subsection{Subjects}

Three patients who underwent surgery to treat a bone tumor originating in the pelvis at Fukushima Medical University Hospital since 1996 were investigated (Table 1). At the time of surgery, two patients were 43 years old and one patient was 66 years old. There were two men and one woman. The patients' histopathological diagnoses were chondrosarcoma, malignant fibrous histiocytoma of bone, and giant cell tumor of bone (GCTB). The disease stages, assessed using the surgical staging system (modified Enneking classification [2] by the MSTS (Musculoskeletal Tumor Society)/ISOLS (International Society of Limb Salvage), were IB and IIB in two patients. The GCTB was locally aggressive.

For the tumor resection, Patients 1 and 2 (who had malignant tumors) underwent a wide resection. Patient 3 (who had GCTB) underwent a marginal resection.

The reconstructive method for each patient was decided based on the extent of bone resection. Patient 1 , who required a resection of the wing of the ilium (Type I [3]) alone, underwent pelvic ring reconstruction with a double-barreled free vascularized fibular graft (FVFG). The FVFG was placed between the sacrum and the iliac bone, and was fixed with a reconstruction plate (Figure 1). Patient 2, who required resection of the iliac bone and acetabular portion (Type I + II), underwent a pelvic ring reconstruction using an FVFG according to the method described by Nagoya et al. [1] and simultaneous hip arthrodesis. The FVFG was placed between the sacrum and femoral head and fixed with a screw. With the use of a spine instrument, the area between the fifth lumbar vertebra and femur was fixed rigidly, and the pelvic ring reconstruction and hip arthrodesis were performed simultaneously. The fixation angle of the hip was $30^{\circ}$ of flexion, and the leg length discrepancy was 2 cm (Figure 2).

In contrast, while Patient 3 had acetabular resection (Type I + II), a free latissimus dorsi flap surgery alone was performed due to extensive coverage of soft tissue defects, and the pelvic ring and hip were consequently not reconstructed (Figure 3).

The postoperative clinical courses of all three patients showed successful engraftment of tissues. Patients 1 and 2 (who received an FVFG) showed bone union of the remaining pelvic ring and the grafted fibula at 3 months postoperatively. Complications such as fracture of the grafted fibula or infection were not observed, and

Table 1. Patients' and operative characteristics.

\begin{tabular}{ccccccc}
\hline Pt. No. & Age (ys)/Sex & $\begin{array}{c}\text { Postoperative } \\
\text { follow-up period }\end{array}$ & Pathological diagnosis & $\begin{array}{c}\text { Stage } \\
\text { (MSTS/ISOLS) }\end{array}$ & $\begin{array}{c}\text { Extent of bone } \\
\text { resection }\end{array}$ & $\begin{array}{c}\text { Graft } \\
\text { tissue }\end{array}$ \\
\hline 1 & $43 / \mathrm{F}$ & $12 \mathrm{y} 7 \mathrm{~m}$ & Chondrosarcoma & IB & I & FVFG \\
2 & 66/M & $12 \mathrm{y} 8 \mathrm{~m}$ & Malignant fibrous histiocytoma of bone & IIB & I/II & FVFG \\
3 & $43 / \mathrm{M}$ & $10 \mathrm{y} 4 \mathrm{~m}$ & Giant cell tumor of bone & 3 (aggressive) & I/II & LD \\
\hline
\end{tabular}

FVFG, free vascularized fibular graft; LD, latissimusdorsi free flap. 


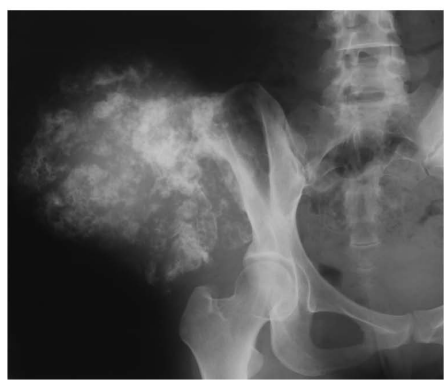

(a)

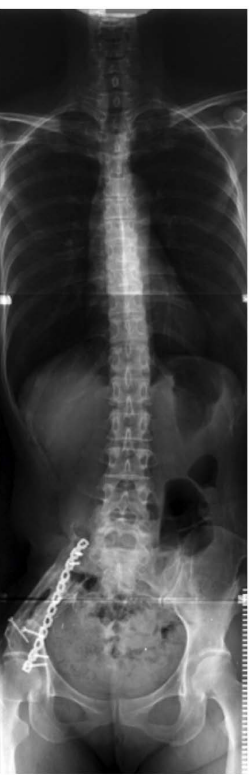

(b)

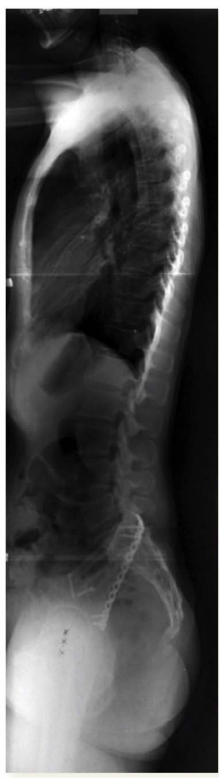

(c)

Figure 1. Patient 1, a 43-year-old woman. Calcification caused by a giant chondrosarcoma was observed at the right iliac bone (a). Whole spine radiography in a standing position, anteroposterior (b) and lateral (c) views. Type I resection was performed, and the pelvic ring was reconstructed using an FVFG. Scoliosis with a Cobb angle of 3 was observed, but the spinal alignment in the sagittal plane was satisfactory.

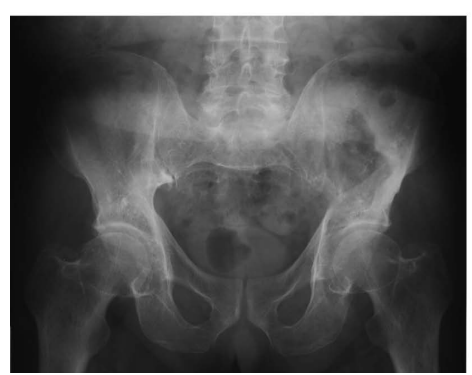

(a)

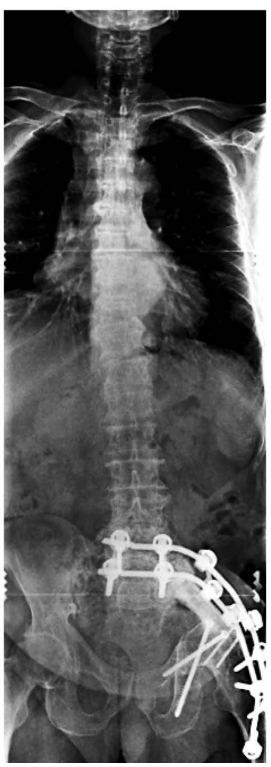

(b)

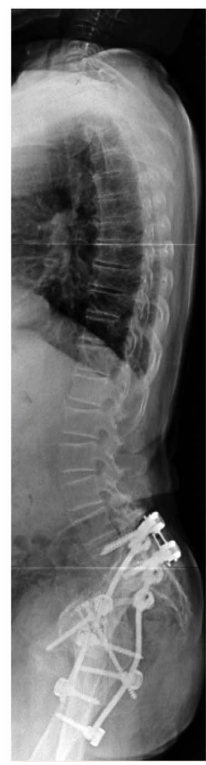

(c)

Figure 2. Patient 2, a 66-year-old man. Bone destruction caused by a malignant fibrous histiocytoma of bone was observed at the left iliac bone to the upper acetabulum (a). Whole spine radiography in a standing position, anteroposterior (b) and lateral (c) views. Using an FVFG and a spine system, pelvic ring reconstruction and hip arthrodesis were performed. The hip fixation angle was $30^{\circ}$ of flexion, and the leg length discrepancy was $2 \mathrm{~cm}$. The anteroposterior view shows mild scoliosis. The lateral view shows an anterior pelvic tilt and anterior tilt balance, but the spinal alignment was satisfactory. 


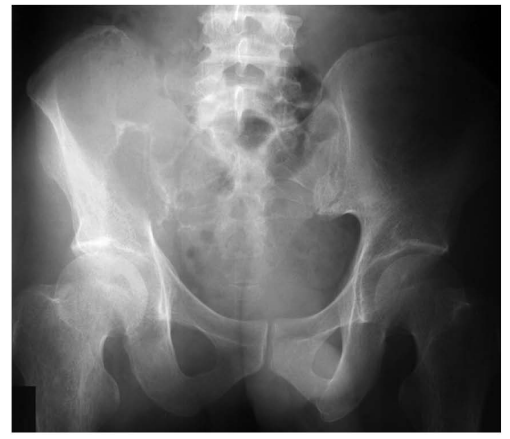

(a)

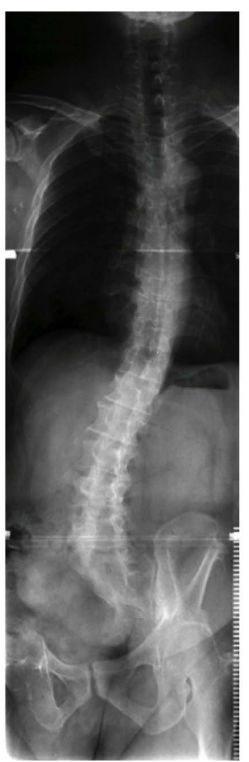

(b)

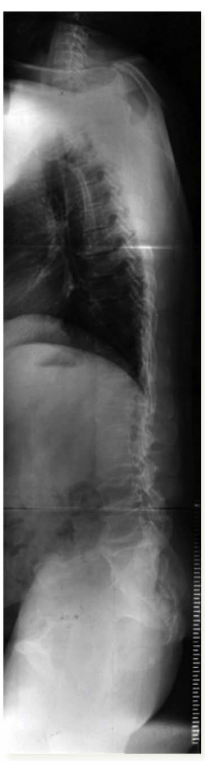

(c)

Figure 3. Patient 3, a 43-year-old man. Bone destruction caused by a giantcell tumor of bone was observed from the sacrum to the right iliac bone (a). Whole spine radiography in a standing position, anteroposterior (b) and lateral (c) views. The sacrum to the right acetabular portion had developed into a flail hip due to extensive resection. The anteroposterior view shows advanced scoliosis. The lateral view shows a loss of physiological curvature of the spine and a marked posterior pelvic tilt.

the grafted fibula showed a satisfactory transverse diameter enlargement over time.

In contrast, Patient 3 (who received a latissimus dorsi free flap alone) did not have a continuous pelvic ring and had a flail hip. From the perspective of trunk support, the pelvic ring instability increased and the hip function decreased in order from Patient 1 to Patient 3.

The post-reconstruction follow-up periods in these three patients were 12 years and 8 months, 12 years and 7 months, and 10 years and 4 months.

\subsection{Evaluation Method}

Spinal deformity and QOL were evaluated in all three patients. Spinal deformity is a secondary change that occurs due to pelvic deformation or hip dysfunction after surgery and is thought to have pathology similar to that of hip-spine syndrome [4]. Therefore, radiological measurement methods [5] [6] that are used to evaluate hipspine syndrome were used to determine spinal deformity (Figure 4). Using whole spine radiography, the following four parameters were measured: the Cobb angle (Figure 4(a)), the thoracic kyphosis angle (TKA) and lumbar lordosis angle (LLA) (Figure 4(b)), the pelvic angle (PA) in the sagittal plane (Figure 4(c)), and spinal balance in the sagittal plane (i.e., the distance from the C7 plumb line to the hip axis: C7 PL-HA) (Figure 4(d)).

The measurements were performed in triplicate by the same examiner, and the means of these measurements were used. The TKA was measured as the angle between the lines extending from the superior edge of the first thoracic vertebra and the inferior edge of the twelfth thoracic vertebra. The LLA was measured as the angle between the lines extending from the inferior edge of the twelfth thoracic vertebra and the superior edge of the sacrum.

For the PA and C7 PL-HA measurements, the midpoint of the line that connects the center points of both the left and right acetabula was set as the hip axis (HA). The angle between the line that connects the HA, the posterosuperior corner of S1, and the plumb line was measured as the PA. The distance between the C7 plumb line (a plumb line from the center point of the body of the seventh cervical vertebra) and the HA was measured as the C7 PL-HA. We compared the patients' TKA, LLA, PA, and C7 PL-HA values with the appropriate levels for Japanese people [7]. 


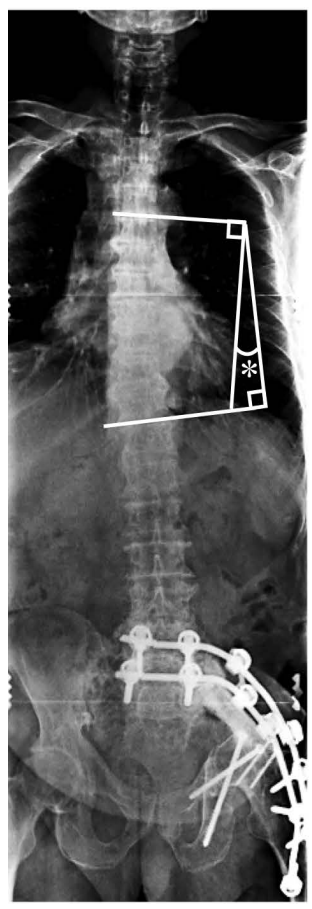

(a)

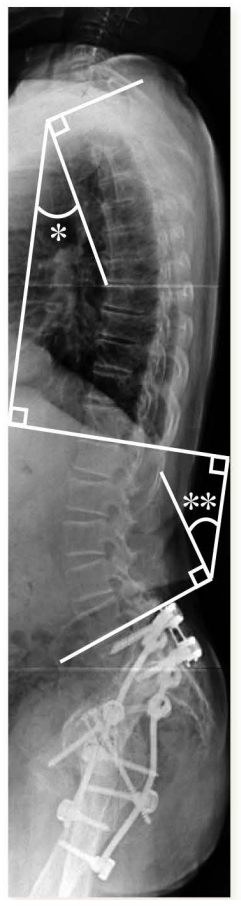

(b)

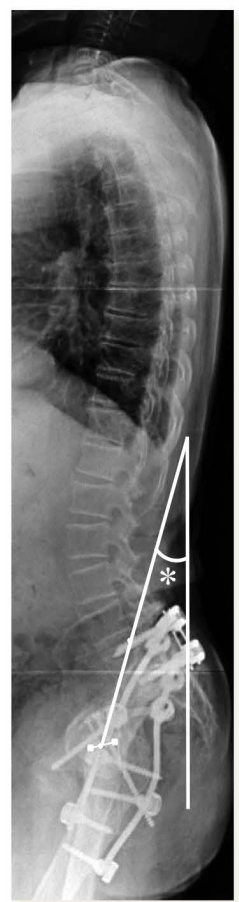

(c)

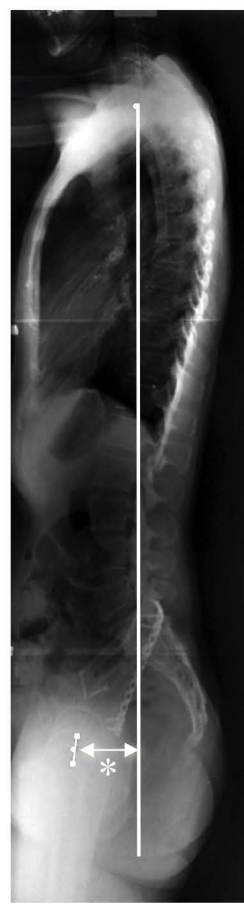

(d)

Figure 4. Spinal deformity measurement methods. (a) * Cobb angle. (b) *Thoracic kyphosis angle (TKA), the angle between the lines extending from the superior edge of Th1 and the inferior edge of Th12. ${ }^{* *}$ Lumbar lordosis angle (LLA), the angle between the lines extending from the inferior edge of Th12 and the superior edge of the sacrum. (c) HA (hip axis), the midpoint of the line that connects the acetabula on both sides. *PA (pelvic angle), the angle between the line that connects the HA and the posterosuperior corner of S1and the plumb line. (d) ${ }^{*} \mathrm{C} 7$ PL-HA, the distance between the plumb line from the center of the C7 vertebral body (C7 plumb line) and the HA (hip axis).

The patients' QOL was assessed with Enneking's functional evaluation of the affected limb (ISOLS score) [3], the Roland-Morris Disability Questionnaire(RDQ), activities of daily living (ADL) satisfaction using a visual analogue scale(VAS), and the SF-36 short-form health survey.

\section{Results}

\subsection{Spinal Deformity (Table 2)}

\subsubsection{Cobb Angle}

The Cobb angles were $3^{\circ}, 11^{\circ}$, and $22^{\circ}$ for Patients 1,2 , and 3 , respectively. These results show that spinal alignment in the frontal plane was exacerbated by pelvic ring disruption and decreased hip function.

\subsubsection{TKA and LLA}

The TKAs were $38^{\circ}, 32^{\circ}$, and $4^{\circ}$ and the LLAs were $49^{\circ}, 44^{\circ}$, and $7^{\circ}$ for Patients 1,2 , and 3 , respectively, indicating that the physiological curvature of the spine decreases with disruption of the pelvic ring and hip.

Compared to the appropriate levels for Japanese people, both the TKA and the LLA deviated from the appropriate level in Patient 3. This indicated that spinal alignment in the sagittal plane can be maintained favorably by reconstructing the pelvic ring and hip durability for weight bearing.

\subsubsection{PA}

The PAs, which represent the extent of pelvic rotation in the sagittal plane, were $-25^{\circ}$ and $-15^{\circ}$ in Patients 1 and 2, respectively, and both were at appropriate levels for Japanese people. In contrast, a posterior pelvic tilt with the PA of $-44^{\circ}$ was observed in Patient 3 , indicating that the pelvic rotation in the sagittal plane can be favorably maintained by reconstructing the pelvic ring and hip durability for weight bearing. 
Table 2. Spinal deformity.

\begin{tabular}{|c|c|c|c|c|c|c|}
\hline Pt. No. & Type of reconstruction & $\begin{array}{c}\text { Cobb angle } \\
\text { (degrees) }\end{array}$ & $\begin{array}{c}\text { TKA } \\
\text { (degrees) }\end{array}$ & $\begin{array}{c}\text { LLA } \\
\text { (degrees) }\end{array}$ & $\begin{array}{c}\text { PA } \\
\text { (degrees) }\end{array}$ & $\begin{array}{c}\text { C7 PL-HA } \\
\text { (mm) }\end{array}$ \\
\hline 1 & Pelvic ring reconstruction & 3 & 38 & 49 & -25 & -40 \\
\hline 2 & Pelvic ring reconstruction and hip arthrodesis & 11 & 32 & 44 & -15 & 17 \\
\hline \multirow[t]{2}{*}{3} & Flail hip & 22 & 4 & 7 & -44 & 19 \\
\hline & Appropriate levels in Japanese people [7] & & $16-64$ & $34-74$ & -5 to -29 & $<0$ \\
\hline
\end{tabular}

TKA, thoracic kyphosis angle; LLA, lumbar lordosis angle; PA, pelvic angle; C7 PL-HA, distance between C7 plump line and hip axis.

\subsubsection{C7 PL-HA}

The C7 PL-HA, which indicates the anteroposterior balance of the trunk in the sagittal plane, is reported [5] as negative when there is appropriate anteroposterior balance, because the C7 plumb line passes through posterior to the HA. Patient 1 had an appropriate C7 PL-HA value, $-40 \mathrm{~mm}$. In contrast, Patients 2 and 3 showed poor anteroposterior balance with C7 PL-HA values of $+17 \mathrm{~mm}$ and $+19 \mathrm{~mm}$, respectively, denoting that not only pelvic ring disruption, but also the presence or absence of hip arthrodesis affects sagittal plane balance.

\subsubsection{ISOLS Score (Table 3)}

The ISOLS score [3] in Patient 1, who underwent only pelvic ring reconstruction, was satisfactory at 93\%. Low ISOLS scores were obtained by Patients 2 and 3. Patient 2, who additionally underwent hip arthrodesis, had a score of 53\%, and Patient 3 who did not undergo pelvic ring reconstruction had a score of $23 \%$. These results showed that both pelvic ring reconstruction and hip arthrodesis affect postoperative lower extremity function.

\subsubsection{Roland-Morris Disability Questionnaire (RDQ) Score (Table 4)}

Patient 1's score on the RDQ, a low back pain-specific QOL measurement, was higher than the national norm, whereas the scores of the other two patients were lower than the national norm. This implied that hip arthrodesis and pelvic ring disruption can elicit low back pain that could decrease QOL.

\subsubsection{ADL Satisfaction (Table 4)}

ADL satisfaction using the VAS was high in both Patients 1 and 2, with scores of 100\% and 80\%, respectively. In contrast, Patient 3 had a markedly low score of 15\%. Thus, for subjective ADL improvement, pelvic ring reconstruction and hipsupport are essential.

\subsubsection{SF-36 (Table 5)}

For the SF-36, which measures health-related QOL, Patient 1 had higher scores compared to the national normin all subscales. In contrast, the other two patients had scores lower than the national norm. In particular, Patient 3 (with pelvic ring disruption) had low scores in physical functioning and in both the role-physical and role-emotional subscales. These results demonstrated that hip arthrodesis and pelvic ring disruption negatively impact both physical and mental health.

\section{Discussion}

Various methods of pelvic ring reconstruction after pelvic tumor resection have been reported. Reconstruction with mega prostheses [8] are known to be superior in early postoperative support but have risks of deep infection, as well as long-term loosening and damage. Allogeneic bone grafts [9] have also been reported to present risks of pseudoarthrosis, fracture, and infection, and are difficult to obtain in Japan. In recent years, although reconstructions using autogenous bone grafts after intraoperative extracorporeal heat treatment [9] or irradiation [10] have been reported, pseudarthrosis, fracture, and infection are issues, as they are for allogeneic bone grafts. Since pelvic ring reconstruction with an FVFG [1] uses living bone, an enhancement of the rate of bone union, the prevention of infection, and the assurance of strength due to transverse diameter enlargement can be anticipated in comparison to the aforementioned reconstruction method. In addition, by using a double-barreled technique, support can also be reinforced. Indeed, in two of the present patients, bone union was achieved within 3 postoperative months; infections or fractures were not observed, and long-term rigid stability was maintained. 
Table 3. ISOLS score [3].

\begin{tabular}{|c|c|c|c|c|c|c|c|c|}
\hline Pt. No. & Type of reconstruction & Pain & Function & $\begin{array}{l}\text { Emotional } \\
\text { acceptance }\end{array}$ & Supports & $\begin{array}{l}\text { Walking } \\
\text { ability }\end{array}$ & Gait & $\begin{array}{c}\text { Overall } \\
\text { rating }(\%)\end{array}$ \\
\hline 1 & Pelvic ring reconstruction & 5 & 5 & 5 & 5 & 5 & 3 & 93 \\
\hline 2 & $\begin{array}{l}\text { Pelvic ring reconstruction and } \\
\text { hip arthrodesis }\end{array}$ & 5 & 5 & 3 & 0 & 3 & 1 & 57 \\
\hline 3 & Flail hip & 3 & 0 & 3 & 0 & 1 & 0 & 23 \\
\hline
\end{tabular}

Table 4. RDQ scores and ADL satisfaction.

\begin{tabular}{clcc}
\hline Pt. No. & Type of reconstruction & RDQ score & ADL satisfaction \\
\hline 1 & Pelvic ring reconstruction & 58.0 & 100 \\
2 & Pelvic ring reconstruction and hip arthrodesis & $30.6^{*}$ & 80 \\
3 & Flail hip & $19.7^{*}$ & 15 \\
\hline
\end{tabular}

RDQ: Roland-Morris Disability Questionnaire; ADL: activities of daily living. * Less than the Japanese national norm (50 points).

Table 5. SF-36 scores.

\begin{tabular}{clcccccccc}
\hline Pt. No. & Type of reconstruction & PF & RP & BP & GH & VT & SF & RE & MH \\
\hline 1 & Pelvic ring reconstruction & 51.6 & 52.8 & 61.4 & $48.9^{*}$ & 56.4 & 57.1 & 56.6 & 54.4 \\
2 & Pelvic ring reconstruction and hip arthrodesis & $48.1^{*}$ & $29.0^{*}$ & $35.3^{*}$ & $38.1^{*}$ & $47.2^{*}$ & $30.8^{*}$ & $31.1^{*}$ & $49.1^{*}$ \\
3 & Flail hip & $-4.7^{*}$ & $1.7^{*}$ & $30.9^{*}$ & $46.2^{*}$ & $47.2^{*}$ & $37.4^{*}$ & $18.3^{*}$ & 51.8 \\
\hline
\end{tabular}

PF, physical functioning; RP, role-physical; BP, bodily pain; GH, general health; VT, vitality; SF, social functioning; RE, role-emotional; MH, mental health; " Less than the Japanese national norm (50 points).

After pelvic tumor resection, varying degrees of morphological and mechanical disruptions occur to the pelvis and hip depending on the extent of resection and the presence or absence of reconstruction. These changes elicit long-term compensatory alignment abnormalities to the spine with the adjacent weight-bearing joint. This is thought to be a pathology similar to that of secondary hip-spine syndrome [4]. Therefore, in the present study, we investigated spinal deformity by the measurement method used to evaluate hip-spine syndrome.

With regard to the spinal alignment in the frontal plane, the results of the present study revealed that the extent of scoliosis differs depending on the presence or absence of pelvic ring reconstruction and hip arthrodesis, even in cases with acetabular resection. This suggested that, if hip durability for weight bearing can be reconstructed as it was in Patient 2, secondary scoliosis can be reduced.

Regarding alignment in the sagittal plane, only Patient 3 (who did not undergo pelvic ringer construction and hip arthrodesis) had physiological curvature of the spine and pelvic rotation that deviated from the Japanese national norm. This indicated that it is possible to maintain satisfactory spinal alignment in the sagittal plane by reconstructing the pelvic ring and fixing the hip in a favorable limb position.

In terms of the spinal balance in the sagittal plane, when the pelvis becomes tilted in the anteroposterior direction, the physiological curvature of the spine typically changes as compensation, such that the sagittal plane balance is maintained at the appropriate level. Patient 3, who did not undergo pelvic ring reconstruction, had poor anteroposterior balance, even though the physiological curvature of his spine had decreased as compensation. This signifies that, when pelvic deformity progresses, anteroposterior balance will not be completely corrected even if there are compensatory spinal alignment changes.

Patient 2 who underwent both pelvic ring reconstruction and hip arthrodesis, also had sagittal plane balance that deviated from the norm. Since Patient 2's hip was fixed at $30^{\circ}$ flexion, the pelvis had an anterior tilt in the standing position compared to the other two patients, and for this reason, the physiological curvature of the spine should normally compensate by curving further. However, in actuality, compared to Patient 1 who had an even smaller anterior pelvic tilt, Patient 2's physiological curvature was conversely reduced. This may have been due to the effects of spine mobility, indicating that because Patient 2 was significantly older than the other two pa- 
tients (78 yrs old vs.55 and 53 yrs old) at the final follow-up and because spondylosis-related changes were evident on the plane radiographs, there is a possibility that the compensation was inadequate due to a reduction in spinal mobility.

Regarding the patients' QOL, the ISOLS scores for pain, function, and emotional acceptance [3] were high in Patients 1 and 2. If the pelvic ring is reconstructed and hipstability is achieved, then pain caused by spinal deformity can be avoided, and functionally favorable satisfaction can also be obtained. In contrast, the categories of support, walking ability, and gait showed that Patient 2 had low support and gait scores due to claudication and the use of bilateral crutches during walking. However, compared to Patient 3, who uses a wheelchair regularly due to difficulties in weight-bearing of the affected limb, Patient 2 had clearly higher scores. Even after acetabular resection, walking ability that is helpful in daily life can be gained with hip arthrodesis.

Using the RDQ, we investigated the effects of low back pain (which is induced in conjunction with spinal deformity) on the patients' QOL. Patient 1 (who underwent pelvic reconstruction) did not experience low back pain throughout the entire clinical course. In contrast, despite the spinal deformity itself not being largely different from that of Patient 1, Patient 2 experienced low back pain when he was in a supine position, at the start of movement, and during long periods of standing or walking. Patient 2's RDQ score was accordingly low. Given the results, this could be due to posture abnormality caused by the exacerbation of anteroposterior balance. However, there are several other probable causes of low back pain, such as lordosis exacerbation in the supine position due to the limb position of the hip arthrodesis, or spondylosis-related changes due to aging. As such, it is difficult to ascertain to what extent anteroposterior balance exacerbation alone is involved in the RDQ assessment score.

Patient 3 with advanced spinal deformity had persistent low back pain across all types of ADL movements, and the RDQ score was lower than that of Patient 2. In other words, spinal deformity that is induced in association with pelvic ring disruption can elicit low back pain, which can secondarily decrease QOL.

In terms of the patients' self-evaluated QOL, the VAS score of ADL satisfaction was markedly lower in Patient 3 with pelvic ring disruption. Moreover, the SF-36results also indicated markedly low physical functioning and role-physical (physical role functioning), as well as poor role-emotional (emotional role functioning), in Patient 3. These results showed that the decline in physical health due to pelvic ring disruption can also elicit a decline in mental health.

\section{Summary}

The results of the present study demonstrated that after pelvic tumor resection, not only pelvic ring disruption but also the presence or absence of acetabular resection affect the long-term clinical course, including spinal deformity and QOL. Our findings also clarified that, by performing pelvic ring reconstruction together with hip arthrodesis, the exacerbation of spinal deformity or QOL can be reduced, even in acetabular resection cases. Pelvic ring reconstruction and hip arthrodesis using an FVFG are superior operative procedures that can maintain satisfactory spinal alignment and QOL over a long period of time. The present study is preliminary, investigating only three patients; this is a major limitation. Further studies are needed with larger numbers of patients with pelvic tumors who underwent microsurgical reconstruction after radical resection.

\section{Conflict of Interest Disclosure}

The authors declare that they have no conflict of interests or financial profit related to this study.

\section{References}

[1] Nagoya, S., Usui, M., Ishii, S., et al. (2000) Reconstruction and Limb Salvage Using a Free Vascularised Fibular Graft for Periacetablar Malignant Bone Tumors. Journal of Bone \& Joint Surgery, British Volume, 82, 1121-1124. http://dx.doi.org/10.1302/0301-620X.82B8.11004

[2] Enneking, W.F., Spanier, S.S. and Goodman, M.A. (1980) A System for the Surgical Staging of Musculoskeletal Sarcoma. Clinical Orthopaedics and Related Research, 153, 106-120.

[3] Enneking, W.F., Dunham, W.K., Gebhardt, M.C., et al. (1993) A System of the Functional Evaluation of Reconstructive Procedures after Surgical Treatment of Tumors of the Musculoskeletal System. Clinical Orthopaedics and Related Research, 286, 241-246.

[4] Offierski, C.M.. and MacNab, I. (1983) Hip-Spine Syndrome. Spine, 8, 316-321. 
http://dx.doi.org/10.1097/00007632-198304000-00014

[5] Jackson, R.P., Peterson, M.D., McManus, A.C., et al. (1998) Compensatory Spinopelvic Balance over the Hip Axis and Better Reliability in Measuring Lordosis to the Pelvic Radius on Standing Lateral Radiographs of Adult Volunteers and Patients. Spine, 23, 1750-1767. http://dx.doi.org/10.1097/00007632-199808150-00008

[6] Jackson, R.P. and McManus, A.C. (1994) Radiographic Analysis of Sagittal Plane Alignment and Balance in Standing Volunteers and Patients with Low Back Pain Matched for Age, Sex, and Size. A Prospective Controlled Clinical Study. Spine, 19, 1611-1618. http://dx.doi.org/10.1097/00007632-199407001-00010

[7] Kanemura, T., Satake, K., Yamaguchi, H., et al. (2013) Sagittal Spino-Pelvic Alignment in an Asymptomatic Japanese Population (in Japanese). Orthopaedic Surgery \& Traumatology, 56, 805-814.

[8] Uchida, A., Myoui, A., Araki, N., et al. (1996) Prosthetic Reconstruction for Periacetablar Malignant Tumors. Clinical Orthopaedics, 326, 238-245. http://dx.doi.org/10.1097/00003086-199605000-00029

[9] Harrington, KD. (1992) The Use of Hemipelvic Allografts or Autoclaved Grafts for Reconstruction after Wide Resection of Malignant Tumors of the Pelvis. Journal of Bone \& Joint Surgery, 74, 331-341.

[10] Myoui, A., Araki, N., Ueda, T., et al. (2002) Clinical Results of the Intraoperative Extracorporeal Autogenous Irradiated Bone Graft. Journal of the Japanese Orthopaedic Association, 76, S828. 